\title{
DISCOVERING MEANINGFUL MULTIMEDIA PATTERNS WITH AUDIO-VISUAL CONCEPTS AND ASSOCIATED TEXT
}

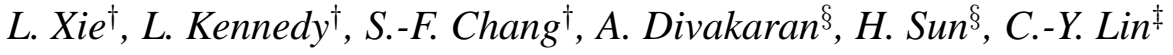 \\ ${ }^{\dagger}$ Dept. of Electrical Engineering, Columbia University, New York, NY \\ ${ }^{\ddagger}$ IBM T.J. Watson Research Center, Hawthone, NY \\ ${ }^{\S}$ Mitsubishi Electric Research Labs, Cambridge, MA
}

\begin{abstract}
The work presents the first effort to automatically annotate the semantic meanings of temporal video patterns obtained through unsupervised discovery processes. This problem is interesting in domains where neither perceptual patterns nor semantic concepts have simple structures. The patterns in video are modeled with hierarchical hidden Markov models (HHMM), with efficient algorithms to learn the parameters, the model complexity, and the relevant features; the meanings are contained in words of the speech transcript of the video. The pattern-word association is obtained via co-occurrence analysis and statistical machine translation models. Promising results are obtained through extensive experiments on 20+ hours of TRECVID news videos: video patterns that associate with distinct topics such as el-nino and politics are identified; the HHMM temporal structure model compares favorably to a non-temporal clustering algorithm.
\end{abstract}

\section{INTRODUCTION}

This paper presents solutions towards discovering meaningful structures in video in multiple modalities. Structures, or patterns, in temporal sequences refer to the repetitive segments that bear consistent characteristics in the observations and the dynamics. Automatic identification of structures from video is an interesting topic for both theoretical problems on learning in multi-modality and applications on multimedia content organization.

Supervised learning techniques are capable of learning the target structure once the domain knowledge is encoded in the training data, the choice of the feature set, the construction of the statistical model, and the design of the learning algorithms. Unsupervised structure discovery, on the other hand, tries to find statistical descriptions of the structures with much less information available. It has been shown [1] that interesting game states in sports videos can be revealed without supervision using a few lowlevel features. Temporal structures identified by pure computational criteria awaits association and evaluation with meanings before they become usable. This association can be manually performed when meanings in the original content are unambiguous and few, such as in sports programs. However, in domains with more general and diverse content, such as news videos, tagging structures with meanings is no longer a straight-forward task, and the difficulty comes from the large number of topics present and the inherent complexity in the large hierarchy of meanings. In produced video content, the prevalence of metadata, such as closed captions, speech transcript, gamestats or screenplays, provides a complimentary channel of semantic information for bridging this gap.
In this work, we aim to discover meaningful structures in audiovisual concept space with the additional information provided in the metadata. An audio-visual concept space is a collection of elementary concepts such as "people", "building", and "monologue" each of which was learned from low-level features in a separate supervised training process [2]. We believe that such mid-level concepts offer a promising direction to revealing the semantic meanings in patterns, since grouping and post-processing beyond the signal level is deemed a vital part for the understanding of sensory inputs [3], and multi-modal perception is no less complicated than perception in individual senses. Temporal structures in the audiovisual sequence, characterized by the strength in each concept, the mutual dependency among concepts and their temporal evolutions, are captured by a hierarchical hidden Markov model (HHMM), learnable with statistical inference techniques such as ExpectationMaximization (EM) and Monte Carlo method. Once a description of temporal structure is in place, the first step towards understanding its meaning is to examine the co-occurrence statistics between the structure labels and words obtained from the metadata such as speech transcripts or screenplays. The co-occurrence statistics are further refined by estimating an underlying generative probability between the labels and the words with machine translation models. These techniques were first proposed by Brown et. al. [4], and later used to associate images blobs with words [5, 6]. The former [5] was set in a context with clean text labels that can be treated as concepts in themselves; while the latter [6] operates on the keyframes in video shots without taking into account the temporal structure. We use news videos as the test domain and find promising associations from the video patterns to distinct topics such as el-nino or politics, we have also demonstrated the advantage of using a dynamic structure model over a plain clustering alternative.

The rest of this paper is organized as follows, Section 2 discusses the unsupervised discovery of video patterns using HHMM, Section 3 presents algorithms for associating the patterns with the speech transcript; Section 4 includes the experiment results on news videos; Section 5 summarizes this work and discusses open issues.

\section{UNSUPERVISED LEARNING OF VIDEO PATTERNS}

Solutions to unsupervised structure discovery address two objectives in one pass: finding a statistical description of the structure and locating the corresponding segments in the sequence. We are interested in models that describe the properties of each individual video unit (frame or shot) as well as the temporal transitions among these units. Distinct appearance and transition patterns ex- 
ist in produced video contents such as TV programs and feature films, and a two-level HHMM is a model with an efficient inference algorithm suitable for this purpose. We use the algorithms described in an ealier work [1] to learn the HHMM; a summary is presented in Sections 2.1 and 2.2 for completeness.

Our feature set consists of the confidence values from twelve concept detectors obtained from the IBM concept detection system in TRECVID evaluations [7, 2]. The confidence scores are obtained by fusing the results of multiple support vector machine (SVM) classifiers applied to the key frame of each shot ${ }^{1}$ in the video. We uniformly quantize the scores into three levels before learning the HHMM. The concepts are \{weather, people, sports, non-studio, nature-vegetation, outdoors, news-subject-face, female speech, airplane, vehicle, building, road\}, selected from the 16 TRECVID-evaluated concepts that have a reported average precision greater than $50 \%$. Using shots as the basic analysis units is advantageous for the news domain, because the production syntax usually produces clear cuts, and the content within a shot is usually consistent. We use this concept space mainly for its availability and performance assurance, while the choice of an optimal concept space or a proper concept lexicon is still an open question.

\subsection{Hierarchical hidden Markov models}

HHMM is a generalization of HMM with hierarchical control structure in the hidden states while also being a specialization of Dynamic Bayesian network (DBN). The parameter set $\Theta$ of a twolevel HHMM consists of within-level and across-level transition probabilities and emission parameters that specifies the distribution of observations conditioned on the state configuration. The model parameters can be estimated efficiently via EM with a complexity $O(T)$, where $T$ is the sequence length. The size of the HHMM state-space represents the number of interesting structures in the data, and it is often desirable to determine the size automatically rather than manually supply a fixed value. Reverse-jump Markov chain Monte Carlo (MCMC) provides an effective computational framework for stochastically searching the space of $\mathrm{HH}-$ MMs of all sizes. Details of this approach are in [1].

\subsection{Maintaining a group of models}

The pattern discovery method above can be used on any feature set - leading to the question of which features are relevant, where relevance refers to agreement among a group of features to represent a target concept. In an unsupervised learning context, the criteria for relevance become relative to the numerous concepts simultaneously present but not explicitly pinpointed in the content. Therefore, given the original $D$-dimensional feature set, we maintain a pool of $M$ models learned over $M$ different feature subsets $\left(M \ll 2^{D}\right.$ ) to further explore the meanings in the clusters. We use mutual information criteria to measure the relevance of features with respect to each other, we then run a clustering algorithm over the mutual information to group the features, followed by redundance removal in each feature group with Markov blanket filtering, as detailed in [1].

\section{ASSOCIATING STRUCTURE WITH METADATA}

The quest for meanings in the temporal structures begins by associating HHMM labels (taking values from a pattern lexicon) to tokens (from a word lexicon) in the metadata stream. The objective of this association is two-fold: tagging structures with meanings and assessing the goodness of them.

\footnotetext{
${ }^{1} \mathrm{~A}$ shot refers to a continuous camera take in both time and space.
}

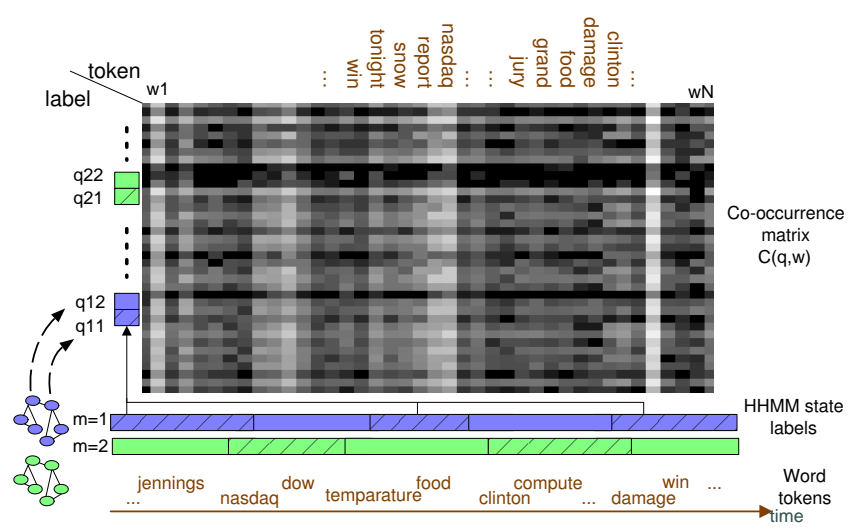

Fig. 1. Generating co-occurrence statistics from the HHMM labels and word tokens.

\subsection{Text processing from speech transcript}

The Automatic Speech Recognition (ASR) transcript of the TREC videos are in the form of time-stamped words $\left(t, \hat{w}_{t}\right)$. The discourse style of a news program is usually concise and direct, hence it suffices to stay at the level of individual words rather than going to higher-level concepts via linguistic or semantic analysis, to represent the subject of discussion. We choose to focus on a lexicon of frequent and meaningful words, freeing ourselves from the noise introduced by stop words and statistically insignificant ones. The lexicon is obtained from the TRECVID development corpus after the following shallow parsing operations: (1) Stem the words from ASR output, resulting in about 12,500 distinct tokens; (2) Prune tokens that appear on average less than once in each half-hour news video, 647 tokens survived; (3) Perform rule-based part-of-speech tagging on the tokens [8], and only retain the 502 nouns, verbs, adjectives or adverbs; (4) Further prune away a few frequent tokens with no concrete meaning such as the verbs "be", "do", "have", the adverbs "more", "even", "just", "still", and words too common in news programs such as "new", "today". Denote the set of pruned ASR tokens from the original transcript as $\left(t, w_{t}\right)$, taking values from the final lexicon $W=\left\{w_{1}, w_{2}, \ldots, w_{N}\right\}$.

\subsection{Co-occurence analysis}

As illustrated in Fig. 1, we obtain the co-occurrence statistic $C(\mathrm{q}, \mathrm{w})$ for a HHMM label $q$ and a token $w$ by counting the number of times that the state label $q$ and the word $w$ both appear in the same temporal segment among all video clips.

Denote the set of $K$ videos as $\mathcal{S}=\left\{\mathcal{S}_{1}, \ldots, \mathcal{S}_{K}\right\}$, let each video $\mathcal{S}_{k}$ be partitioned into a set of closed, non-overlapping segments $\mathrm{S}_{k}=\left\{\mathrm{s}_{i}, i=1, \ldots,\left|\mathrm{S}_{k}\right|\right\}$. Denote the maximum-likelihood state labels on each shot $\bar{s}$ obtained with the HHMMs as $q_{\bar{s}}^{m} \in$ $\mathrm{Q}^{m}, m=1, \ldots, M, \bar{s}=1, \ldots,\left|\bar{S}_{k}\right|, k=1, \ldots, K$, where $m$ indexes the $M$ HHMM models, $\mathrm{Q}^{m}$ is the state-space of the $m$-th HHMM, and $\bar{s}$ indexes the shots in each of the $K$ clips in the current set of videos. The co-occurrence statistic $C\left(\mathrm{q}^{m}, \mathrm{w}\right)$, defined as the number of segments in which both label $\mathrm{q}^{m}$ and token $w$ appear, is accumulated across all the video segments as follows, where " $\bigvee$ " denotes logical $O R$, and 1() is the indicator function.

$C\left(\mathbf{q}^{m}, \mathrm{w}\right)=\sum_{k=1}^{K} \sum_{\mathrm{s} \in \mathrm{S}_{k}} \bigvee_{\substack{\bar{s}=1, \ldots,\left|\bar{S}_{k}\right|, \bar{s} \cap \mathrm{s} \neq \phi}} 1\left(q_{\bar{s}}^{m}=\mathrm{q}^{m}\right) \cdot \bigvee_{t \in \mathrm{s}} 1\left(w_{t}=\mathrm{w}\right)$ 
There are two natural temporal divisions in news videos on which we can compute the co-occurrence statistics: shots $^{1}$ and stories. The latter is defined as "a segment of a news broadcast with a coherent news focus which contains at least two independent, declarative clauses" by TRECVID. Despite the convenience of directly using shots as the temporal division on which the HHMM labels are generated, we find it beneficial to use stories in establishing the label-token correspondence. This is because: (1) Meanings in news are conveyed at the story level with a sequence of shots in the visual channel and several sentences in the speech; the transition in topics happen at story boundaries. (2) Within a shot, words being said are often not in sync with the visual content. Evaluations show that co-occurrence on shots yields precisions only about one-tenth of that of the stories while producing comparable recalls. Without overloading the notations, the set of all stories in video $\mathcal{S}_{k}$ is denoted as $\mathrm{S}_{k}=\left\{\mathrm{s}_{i}, i=1, \ldots, S_{k}\right\}$ hereafter.

Once the co-occurrence statistics are in place, we normalize the co-occurrence counts to obtain the empirical conditional probabilities of tokens and labels, and use these quantities as a basis for "predicting words" in a new video clip. Since the normalization is done within the same HHMM model $m$, we omit the superscript ${ }^{m}$ in the following sections when there is no confusion.

$c\left(\mathbf{w} \mid \mathbf{q}^{m}\right)=\frac{C\left(\mathbf{q}^{m}, \mathbf{w}\right)}{\sum_{\mathrm{w}} C\left(\mathbf{q}^{m}, \mathbf{w}\right)}, \quad c\left(\mathbf{q}^{m} \mid \mathbf{w}\right)=\frac{C\left(\mathbf{q}^{m}, \mathbf{w}\right)}{\sum_{\mathbf{q}^{m}} C\left(\mathbf{q}^{m}, \mathbf{w}\right)}$

\subsection{Refining association with machine translation}

In co-occurrence analysis, we associated all the labels with all the tokens present in the same story, this actually causes many entries $C(\mathrm{q}, \mathrm{w})$ to receive more counts than they "deserve". Take an ideal example, if label $q_{1}$ and token $w_{1}, q_{2}$ and $w_{2}$ always occur and only occur at exactly the same time, respectively; then for each story that contains both $\mathrm{q}_{1}$ and $\mathrm{q}_{2}$, the entries $C\left(\mathrm{q}_{1}, \mathrm{w}_{2}\right)$ and $C\left(\mathrm{q}_{2}, \mathrm{w}_{1}\right)$ will receive one extra count. In other words, we would like to reduce the smoothing effects of token and label correlation resulting from the imprecise association within each story.

It turns out that we cannot just undo the correlation in both dimensions of $C$ simultaneously, however the conditional co-occurrence $c(\mid)$ can be sharpened assuming independence of the variable being conditioned on. A mathematical model for this type of unsmoothing from co-occurrences has been explored in the machine translation (MT) literature [4], where the correspondence from English word e to a French word $f$ are recovered from aligned sentences in both languages, by estimating the translation probabilities $t(f \mid e)$ of $f$ given e, independent of the position of the words. In our context, it is appropriate to estimate both conditionals $t\left(\mathrm{w} \mid \mathrm{q}^{m}\right)$ and $t\left(\mathbf{q}^{m} \mid \mathbf{w}\right)$, as there is no obvious independence in either labels or tokens. Moreover, we do not want to lose the association to either labels or tokens as many entries in $t(\mid)$ diminishes to zero in the estimation process. The translation parameters are estimated with the EM algorithm, which we present from model 1 by Brown et. al. [4] for completeness:

$$
\begin{aligned}
& \mathbf{E}: \quad \bar{t}(\mathbf{q} \mid \mathbf{w})=\frac{t(\mathbf{w} \mid \mathbf{q})}{\sum_{\mathbf{q}} t(\mathbf{w} \mid \mathbf{q})}, \bar{t}(\mathbf{w} \mid \mathbf{q})=\frac{t(\mathbf{q} \mid \mathbf{w})}{\sum_{w} t(\mathbf{q} \mid \mathbf{w})} \\
& \mathbf{M}: \quad t(\mathbf{w} \mid \mathbf{q}) \leftarrow \frac{C(\mathbf{q}, \mathbf{w}) \bar{t}(\mathbf{q} \mid \mathbf{w})}{\sum_{\mathrm{w}} C(\mathbf{q}, \mathbf{w}) \bar{t}(\mathbf{q} \mid \mathbf{w})}, t(\mathbf{q} \mid \mathbf{w}) \leftarrow \frac{C(\mathbf{q}, \mathbf{w}) \bar{t}(\mathbf{w} \mid \mathbf{q})}{\sum_{\mathbf{q}} C(\mathbf{q}, \mathbf{w}) \bar{t}(\mathbf{w} \mid \mathbf{q})}
\end{aligned}
$$

\section{EXPERIMENTS}

In this section, we discuss the results of predicting the correspondence using the co-occurrence statistic and refined probabilities. Our dataset are $\mathrm{ABC}$ and $\mathrm{CNN}$ news broadcasts taken from the
TRECVID 2003 corpus, consisting of 22 half-hour clips from each channel. Each video comes with the audio-visual stream, the ASR words, and the ground-truth for story boundaries. We divide the data into four 11-broadcast sets from either station and rotate their roles as the training set and the test set. The cross-channel testing results are notably worse than that of the same channel, therefore we only report the latter due to space limitations.

We learn HHMMs on one of the 11-video sets (the training set), we maintain $K=10$ different models using different subset of the 12 concepts. We use hierarchical agglomerative clustering on mutual information (Sec. 2.2) to generate feature subsets; the number of models is set to traverse into considerable depth into the clusters; the resulting HHMMs typically have $5 \sim 10$ distinct states. The correspondence of the state labels in all models to the word-stems in the ASR transcript (in the training set) is then estimated according to Equations (2)(3) to produce conditional confidence values $c(\mathrm{w} \mid \mathrm{q}), c(\mathrm{q} \mid \mathrm{w})$ and $t(\mathrm{w} \mid \mathrm{q}), t(\mathrm{q} \mid \mathrm{w})$, respectively. These probabilities can be interpreted in two complementary contexts. One is auto-annotation, i.e., predicting words upon seeing an HHMM label, $c(\mathbf{w} \mid \mathbf{q})$ is the precision value of this token-prediction process on the testing set by the counting processing in Equation (1); the other is retrieval, i.e., producing possible labels upon seeing a word, and $c(\mathbf{q} \mid \mathbf{w})$ is recall of this labelretrieval process. It is easy to see, however, that the precision is biased towards frequent tokens or infrequent labels, while the recall tends to be large with infrequent tokens or frequent labels.

Hence we examine an alternative measure to trade-off this bias: the likelihood ratio $L$ of the estimated conditional to the prior probability. i.e.,

$$
\begin{gathered}
L_{\mathrm{w}}^{c}=c(\mathrm{w} \mid \mathrm{q}) / P(\mathrm{w}), L_{\mathrm{q}}^{c}=c(\mathrm{q} \mid \mathrm{w}) / P(\mathrm{q}) ; \\
L_{\mathrm{w}}^{t}=t(\mathrm{w} \mid \mathrm{q}) / P(\mathrm{w}), L_{\mathrm{q}}^{t}=t(\mathrm{q} \mid \mathrm{w}) / P(\mathrm{q}) ;
\end{gathered}
$$

Where $P(\mathrm{q})$ and $P(\mathrm{w})$ are the empirical fraction of stories that have label q or token $\mathrm{w}$, respectively. Note $L_{\mathrm{w}}^{c}=L_{\mathrm{q}}^{c}$ from the normalizing relationship in Equation 2, and we denote both as $L^{c}$ hereafter. This likelihood ratio measure is essentially the widely used $t f \cdot i d f$ measure in text processing [9], since we are penalizing frequent labels or tokens by scaling with their inverse story frequency. Intuitively, $L \gg 1$ implies a strong association, and $L \rightarrow 0$ implies a stong exclusion, while values close to 1 implies no better than random guess.

We sort all the (label, token) pairs based on $L^{c}, L_{\mathrm{w}}^{t}$ and $L_{\mathrm{q}}^{t}$, respectively, we then examine the salient pairs that lie in the top $5 \%$ of each $L$ values. Table 1 shows details of one interesting label identified. This is the first label (among a total of seven) in a model learnt over the concepts \{outdoors, news-subject-face, building\}. The HHMM feature emission probabilities for this label shows low probability for the concept outdoors and high probability for the other two. The word-level precision and recall are around $20 \%$ and $30 \sim 60 \%$, respectively; the list of words intuitively indicate the topic of politics and legal affairs, the audio-visual content of which often contains scenes of news-subjects and buildings; and the word list is effectively expanded by MT. Further examining the actual stories that contain this label (42 out of a total 216), we find that these 42 stories cover the Iraqi weapon inspection topic with $25.5 \%$ recall and $15.7 \%$ precision, and simultaneously contain the Clinton-Jones lawsuits with $44.3 \%$ recall and $14.3 \%$ precision at the shot-level.

Another interesting label is $q^{6}=3$, indicating high confidence in both of its raw concepts \{people, non-studio-setting $\}$. With a word-list of storm, rain, forecast, flood, coast, el, nino, admin- 


\begin{tabular}{|c|l|c|c|c|c|}
\hline$L^{c} L_{w}^{t} L_{q}^{t}$ & token & $\begin{array}{c}\text { prec. } \\
c(\mathrm{w} \mid \mathrm{q})\end{array}$ & $\begin{array}{c}\text { recall } \\
c(\mathrm{q} \mid \mathrm{w})\end{array}$ & $t(\mathrm{w} \mid \mathrm{q})$ & $t(\mathrm{q} \mid \mathrm{w})$ \\
\hline$\bullet \bullet \bullet$ & murder & 0.095 & 0.571 & 0.028 & 1.000 \\
$\bullet \bullet \bullet$ & lewinski & 0.238 & 0.556 & 0.074 & 0.999 \\
$\bullet \bullet \bullet$ & congress & 0.119 & 0.556 & 0.032 & 0.985 \\
$\bullet \circ \bullet$ & alleg & 0.143 & 0.545 & 0.026 & 0.990 \\
$\bullet \bullet \bullet$ & juri & 0.167 & 0.500 & 0.055 & 1.000 \\
$\bullet \circ \bullet$ & judg & 0.048 & 0.500 & 0.003 & 0.573 \\
$\bullet \circ \bullet$ & clinton & 0.310 & 0.500 & 0.059 & 0.994 \\
$\bullet \bullet \bullet$ & presid & 0.452 & 0.475 & 0.179 & 0.999 \\
$\bullet \circ \bullet$ & polit & 0.167 & 0.467 & 0.031 & 0.972 \\
$\bullet \bullet \bullet$ & saddam & 0.143 & 0.462 & 0.063 & 1.000 \\
$\bullet \bullet \bullet$ & lawyer & 0.143 & 0.462 & 0.039 & 0.999 \\
$\bullet \circ \bullet$ & independ & 0.190 & 0.444 & 0.031 & 0.980 \\
$\bullet \circ \circ$ & accus & 0.095 & 0.444 & 0.000 & 0.010 \\
$\bullet \circ \bullet$ & monica & 0.167 & 0.438 & 0.024 & 0.915 \\
$\bullet \circ \bullet$ & white & 0.381 & 0.432 & 0.061 & 0.876 \\
$\circ \circ \bullet$ & charg & 0.190 & 0.381 & 0.055 & 0.998 \\
$\circ \bullet \bullet$ & investig & 0.167 & 0.412 & 0.055 & 1.000 \\
$\circ \circ \bullet$ & offic & 0.190 & 0.364 & 0.006 & 0.581 \\
$\circ \circ \bullet$ & public & 0.143 & 0.300 & 0.046 & 0.901 \\
$\circ \circ \bullet$ & secretari & 0.190 & 0.364 & 0.019 & 0.689 \\
$\circ \bullet \bullet$ & washington & 0.262 & 0.355 & 0.092 & 0.995 \\
\hline
\end{tabular}

Table 1. Statistics of words associated with label $q^{9}=1$ before and after MT. Note "•" denotes that the corresponding $L$ value lies in the overall top $5 \%$, and "O" is for the complement. Using set A of channel ABC.

istr, water, cost, weather, protect, starr, north, plane, northern, attornei, california, defens, feder, gulf $\}$, it clearly indicates the topic weather. In fact, it covers the el-nino and storms that prevailed the United States in the spring of 1998 with $80 \%$ and $78 \%$ recall on the training and testing set, respectively. Note this weather cluster is found without either the original "weather" concept or any dedicated weather sections in ABC News.

Figure 2 compares the likelihood ratio $L^{c}$ of the HHMMs and the K-means clustering, the latter uses the feature set and cluster size chosen by the HHMM (Sec. 2). From the graph we can see much more strong associations (bright peaks) and exclusions (dark valleys) in the labels obtained with HHMM than that of the $\mathrm{K}$-means, and this shows that temporal modeling is indeed effective for the news domain. Look further into the K-means clusters, take the afore-mentioned \{people, non-studio-setting $\}$ for example, each of the six cluster labels is more spread out across all kinds of stories (appears in at least 2/3 of all stories), which makes its association with topics less distinctive.

It's worth noting that: Words $\neq$ meanings. While the words associated with a few labels are easy to decipher, most labels are associated with diverse words from which distinct topics are difficult to find. Natural language processing techniques such as latent semantic analysis can be employed to unveil the inherent structure in text (e.g., "white" and "house" often appear together) and to embody the ambiguity of going from words to semantics (e.g., "rise" can refer to the stock index, the temperature, a higher elevation, or even an angry political reaction). Furthermore, the concepts in the audio-visual stream may not be those present in the speech transcript, unlike the sentence-wise aligned bitext between two languages [4].

\section{CONCLUSION}

In this paper, we propose a method for discovering meaningful structures in video through unsupervised learning of temporal clusters and associating them with metadata using co-occurrence analysis and models similar to machine translation. We are able to find a few convincing translations between state labels and words in the news domain. We have also observed that temporal models are indeed better at capturing the semantics than non-temporal

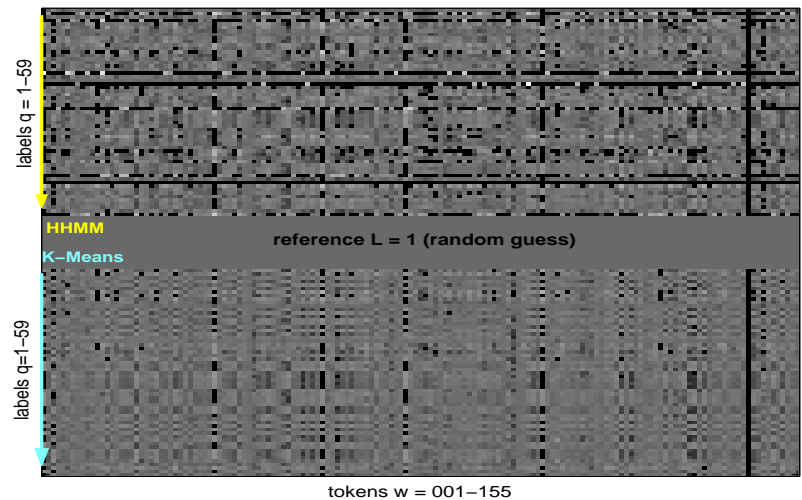

Fig. 2. Likelihood ratios $\log L^{c}(\mathbf{q}, \mathbf{w})$ for HHMM (top) and K-means (bottom). Using set $\mathrm{A}$ of channel $\mathrm{ABC}$.

clustering.

A few interesting issues remain: (1) Using text processing techniques to exploit the correlations inherent in raw word tokens; (2) Joint learning of the temporal model and the semantic association to obtain more meaningful labels.

\section{ACKNOWLEDGEMENT}

We would like to thank the TRECVID team [2] at IBM T. J. Watson Research Center for the concept detection results, and M. Naphade, A. Natsev, and B. Tseng for providing additional visual concept detection results. We are grateful to W. Hsu and D. Zhang for the tokenized speech transcript, the groundtruth for five salient news threads and helpful discussions.

\section{REFERENCES}

[1] L. Xie, S.-F. Chang, A. Divakaran, and H. Sun, Unsupervised Mining of Statistical Temporal Structures in Video, in Video Mining, Kluwer Academic Publishers, 2003.

[2] A. Amir, G. Iyengar, C.-Y. Lin, C. Dorai, M. Naphade, A. Natsev, C. Neti, H. Nock, I. Sachdev, J. Smith, Y. Wu, B. Tseng, and D. Zhang, "The IBM semantic concept detection framework," in TREC Video Retrieval Evaluation Workshop, 2003.

[3] D. Marr, Vision. A Computational Investigation into the Human Representation of Visual Information. New York: Freeman, 1982.

[4] P. F. Brown, S. A. D. Pietra, V. J. D. Pietra, and R. L. Mercer, "The mathematics of statistical machine translation: Parameter estimation," Computational Linguistics, vol. 19, pp. 263311, June 1993.

[5] P. Duygulu, K. Barnard, N. de Freitas, and D. A. Forsyth, "Object recognition as machine translation: Learning a lexicon for a fixed image vocabulary," in ECCV, 2002.

[6] P. Duygulu and H. Wactlar, "Associating video frames with text," in Multimedia Information Retrieval Workshop, in conjuction with SIGIR 2003, (Toronto, Canada), August 2003.

[7] The National Institute of Standards and Technology (NIST), "TREC video retrieval evaluation," 2001-2004. http://wwwnlpir.nist.gov/projects/trecvid/.

[8] H. Liu, "MontyTagger: Commonsense-informed part-ofspeech tagging.", web.media.mit.edu/̌hugo/montytagger/.

[9] D. Jurafsky and J. H. Martin, Speech and Language Processing. Prentice Hall, 2000. 